\title{
Study on axial-flow hydrocyclone for in-situ sand removal of natural gas hydrate in the subsea
}

\author{
Haitao Lin ${ }^{1,2}$, Yuan Huang ${ }^{2,3 *}$ and Hualin Wang ${ }^{2}$ \\ ${ }^{1}$ School of Mechanical and Power Engineering, East China University of Science and Technology, Shanghai, 200237, China \\ ${ }^{2}$ National Engineering Laboratory for Industrial Wastewater Treatment, East China University of Science and Technology, 200237, China \\ ${ }^{3}$ School of Environmental and Chemical Engineering, Shanghai University, Shanghai, 200444, China
}

\begin{abstract}
Natural gas hydrate $(\mathrm{NGH})$ has become the most potential emerging green energy known in the 21 st century due to its characteristics of wide distribution, abundant reserves and clean combustion. This study designs an axial annulus in situ hydrocyclone desander (AAIHD) based on drilling instruments in order to resolve the serious problem of sand production during solid fluidization of NGH. The effect of the inlet flow rate and separation zone length on the sand removal efficiency of the AAIHD is tested through experimental research. The results indicate that AAIHD has a higher separation performance when the separation zone length is $L / D=12.4$ and the inlet flow rate is in the range of $10 \mathrm{~m}^{3} / \mathrm{h}$ to $25 \mathrm{~m}^{3} / \mathrm{h}$, and the maximum separation efficiency reaches $77.4 \%$. The purpose of this study is to achieve in-situ sand removal and the backfilling of sand slurry in addition to facilitate the advancement of solid fluidized exploration technologies.
\end{abstract}

\section{Introduction}

Natural gas hydrate (NGH) is an icy, clathrate compound formed by water and natural gas in a high-pressure and low-temperature environment. NGH is known for its wide distribution, abundant reserves and clean combustion [1]. According to statistics, approximately $99 \%$ of the NGH reserves found in the current surveys are situated in marine sediments on the continental margins [2]. Several hydrate exploiting methods have been proposed in recent years [3], such as depressurization, thermal excitation, chemical injection and $\mathrm{CO}_{2}$ replacement. However, in the exploitation of $\mathrm{NGH}$ in shallow layers of the deep sea, these methods have many problems. One of the key issues is the destruction of the pressure and temperature balance in hydrate reservoirs [4], causing marine geological environmental disasters. The theory of solid fluidization mining was first proposed by Zhou in 2014 for shallow non-diagenetic NGH reservoirs in deep sea environment [5]. However, according to the results of solid fluidization test production in 2017 , it is necessary to separate and backfill a large amount of silty sand in the produced fluid.

In this paper, an axial annulus in-situ hydrocyclone desander (AAIHD) suitable for long and narrow spaces under the seabed is designed based on drilling tools. The purpose of this research is to test the efficiency of a novel type hydrocyclone for in-situ sand removal and assess the feasibility of applying this series of hydrocyclones for sand pretreatment during solid fluidization of $\mathrm{NGH}$ in shallow layers of the deep sea. This study does not involve the separation of hydrates and may be conducted in subsequent studies.

\section{Experiment}

\subsection{Experimental setup and process}

The experimental material is quartz sand with a bulk density of $2650 \mathrm{~kg} / \mathrm{m}^{3}$ and a median particle size of 63.9 $\mu \mathrm{m}$. The volume concentration of quartz sand configured in the experiment is $1 \%$. The in-situ desanding experimental device and process is shown in figure 1 (a). In AAIHD, most of the solid particles in the sand slurry are separated under the action of centrifugal force, discharged from the sand outlet to the casing and return to the storage tank; while the water and a small part of the solid particles are discharged from the NGH outlet to the casing and return to the storage tank. The experimental conditions are inlet flow rates $\left(Q_{i}=10,15,20,25\right.$ and 30 $\left.\mathrm{m}^{3} / \mathrm{h}\right)$ and separation zone lengths $(L / D=3.6,8.0$ and 12.4).

${ }^{*}$ Corresponding author's e-mail: yuanhuang@ecust.edu.cn 
(a)

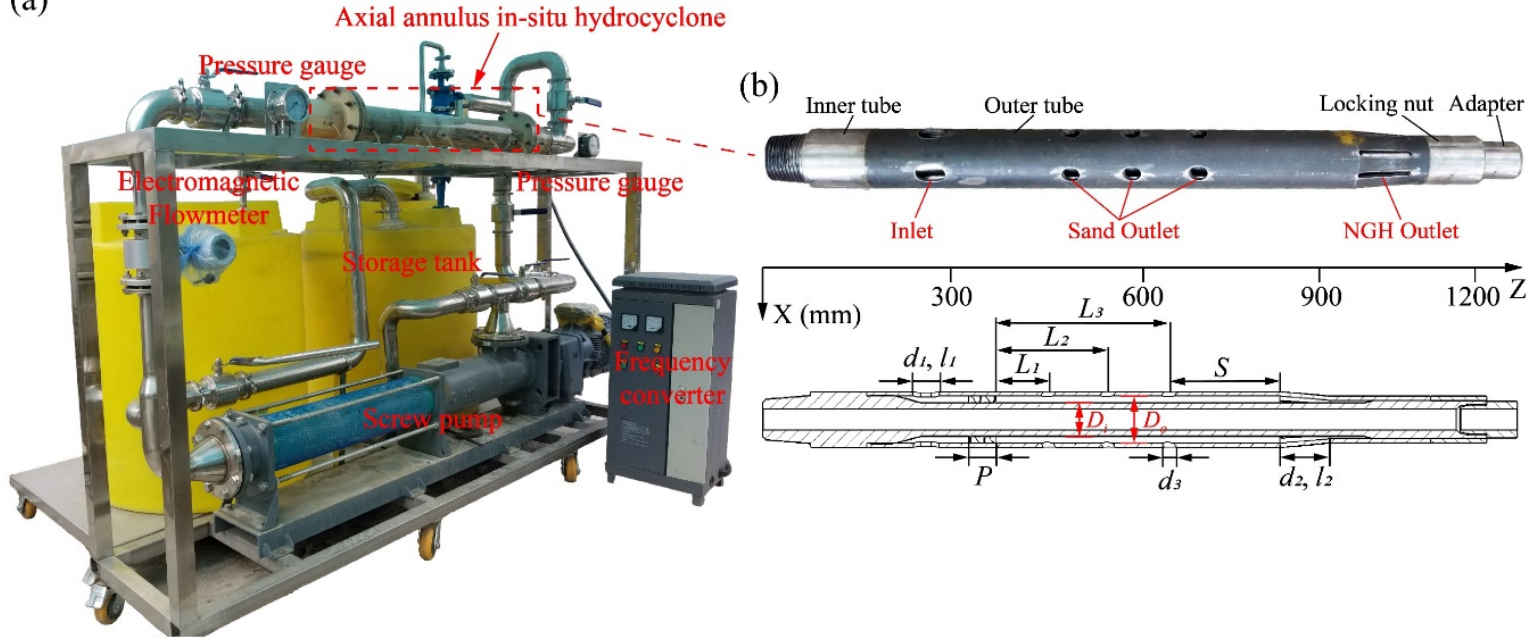

Figure 1. (a) In-situ desanding experimental device and process; (b) the structure of the AAIHD.

The hydrocyclone (AAIHD) designed in the in-situ desanding experiment is shown in figure 1 (b). AAIHD consists of four parts: inner tube, outer tube, locking nut and adapter. On the AAIHD, there is one row of inlets on the AAIHD, three rows of sand outlets and one row of NGH outlets. The three rows of sand outlets respectively correspond to different separation zone lengths $L$. During the experiment, one row of sand outlets is left, and the remaining rows are plugged with hole plugs. The detailed sizes of the AAIHD are shown in table 1, and the hydraulic diameter $D$ of the AAIHD is $25 \mathrm{~mm}$.

Table 1. Dimensions of the AAIHD.

\begin{tabular}{cc|cc}
\hline Parameter & Value $/ \mathrm{mm}$ & Parameter & Value $/ \mathrm{mm}$ \\
\hline$D_{i}$ & 60 & $l_{2}$ & 80 \\
$D_{o}$ & 85 & $L_{1}$ & 90 \\
$d_{1}$ & 30 & $L_{2}$ & 200 \\
$d_{2}$ & 10 & $L_{3}$ & 310 \\
$d_{3}$ & 25 & $P$ & 50 \\
$l_{1}$ & 20 & $S$ & - \\
\hline
\end{tabular}

\subsection{Separation process and principle}

The structure of the casing and the separation process are shown in figure 2 (a). There are 5 sampling ports on the casing: P1, P2, P3, P4 and P5, which are useful for sampling. The initial sand slurry enters the AAIHD from the inlet. Under the guiding action of the guide vanes, the slurry experiences high-speed rotating motion in the separation chamber, and a centrifugal force field is produced. As shown in figure 2 (b), centrifugal force and radial buoyancy force are the most important factors affecting the separation performance of the AAIHD, and they are both a pair of opposing forces. The resultant force $F$ is the determining factor for the radial migration direction of particles, as follows:

$$
F=F_{c}-F_{b}=\frac{\pi d_{s}^{3}}{6} \cdot \frac{u_{\theta}{ }^{2}}{r} \cdot\left(\rho_{s}-\rho_{l}\right)
$$

where $F_{c}$ and $F_{b}$ are the centrifugal and buoyancy forces, respectively, of the solid particles; $d_{s}$ is the diameter of the solid particles; $u_{\theta}$ is the tangential velocity of the solid particles; $r$ is the revolution radius of the solid particles from the center axis of the AAIHD; $\rho_{s}$ and $\rho_{l}$ are the densities of the solid particles and fluid, respectively. Because the density of quartz sand is higher than that of water. Therefore, equation (1) indicates that the solid particles migrate toward the outer wall of the AAIHD under the action of the radial resultant force, and are finally discharged from the sand outlet, thereby achieving solid-liquid separation.

According to the related literature [6] and equation (1), with increasing inlet flow rate, the tangential velocity of the fluid in the axial-flow hydrocyclone significantly increases, and the radial resultant force on the particles also increases; while with increasing axial displacement, the tangential velocity of the fluid in the axial-flow hydrocyclone gradually decreases, and the radial resultant force on the particles decreases. 


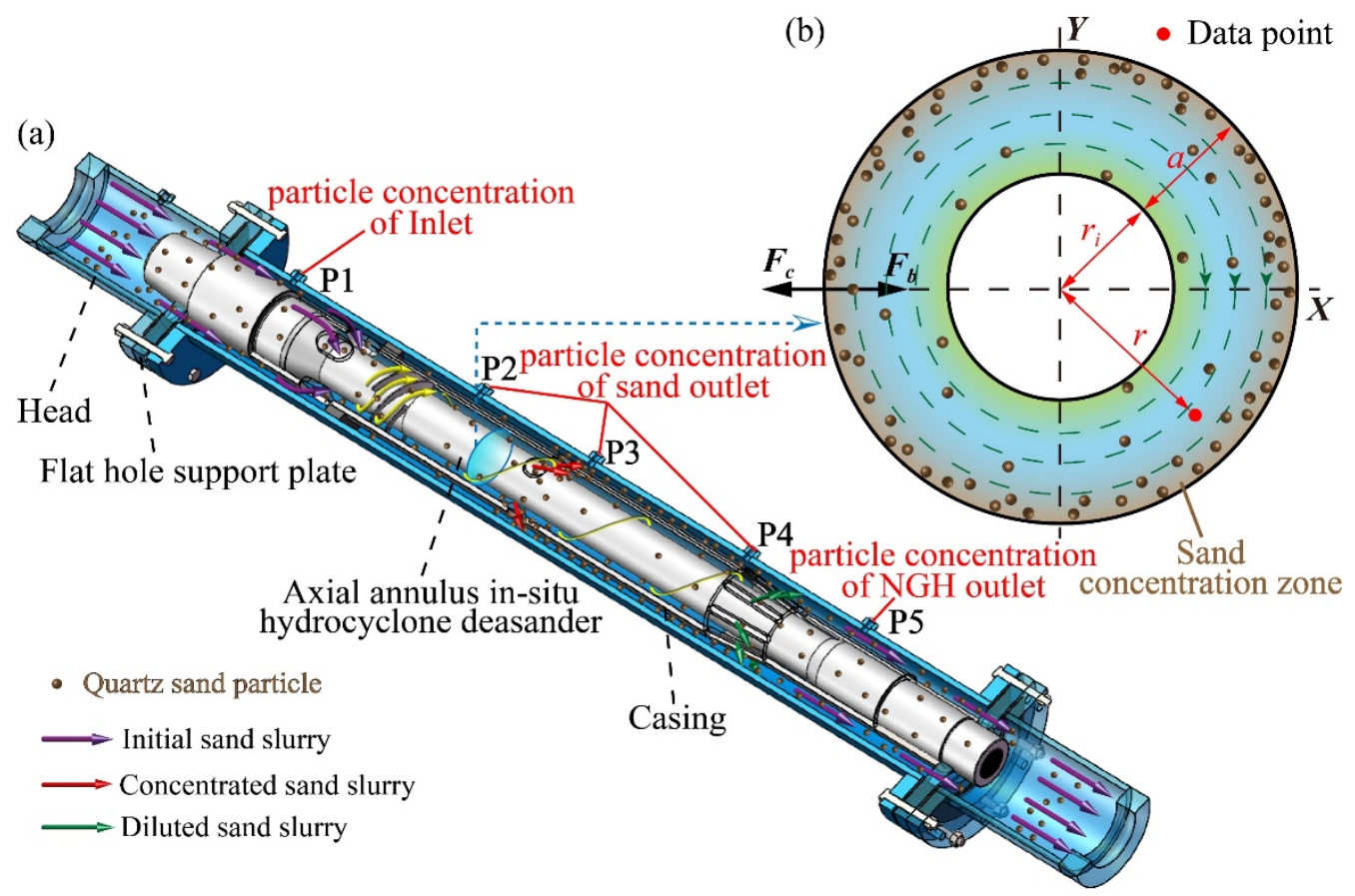

Figure 2. (a) In-situ separation process; (b) separation principle.

\subsection{Average residence time}

In the separation process of the hydrocyclone, the residence time of the dispersed particles in the separation zone is an important factor affecting the separation performance. The average residence time $T_{r}$ of the fluid in the separation zone is defined as follows:

$$
T_{r}=\frac{V_{l}}{Q_{i}}
$$

where $V_{l}$ is the volume of the separation zone, $\mathrm{m}^{3} ; Q_{i}$ is the volume flow rate at the inlet of the AAIHD, $\mathrm{m}^{3} / \mathrm{s}$.

The average residence time $T_{r}$ in the AAIHD under the studied conditions is shown in table 2 .

Table 2. Average residence time.

\begin{tabular}{|c|c|c|c|c|c|}
\hline & & & \multicolumn{3}{|c|}{ Separation zone length $(L / D)$} \\
\hline & & & 3.6 & 8.0 & 12.4 \\
\hline \multirow{5}{*}{$\begin{array}{c}\text { Inlet flowrate } \\
Q_{i}\left(\mathrm{~m}^{3} / \mathrm{h}\right)\end{array}$} & 10 & \multirow{5}{*}{$T_{r}(\mathrm{~s})$} & 0.094 & 0.210 & 0.325 \\
\hline & 15 & & 0.063 & 0.140 & 0.217 \\
\hline & 20 & & 0.047 & 0.105 & 0.163 \\
\hline & 25 & & 0.038 & 0.084 & 0.130 \\
\hline & 30 & & 0.031 & 0.070 & 0.108 \\
\hline
\end{tabular}

\subsection{Split ratio test}

The split ratio $R_{u}$ of the sand outlet is obtained by synchronous high-speed motion analysis system (HSMA), and the high-speed camera velocity measurement process is shown in figure 3. The core part of the HSMA is highspeed camera, LED light source and computer with corresponding professional control software. 


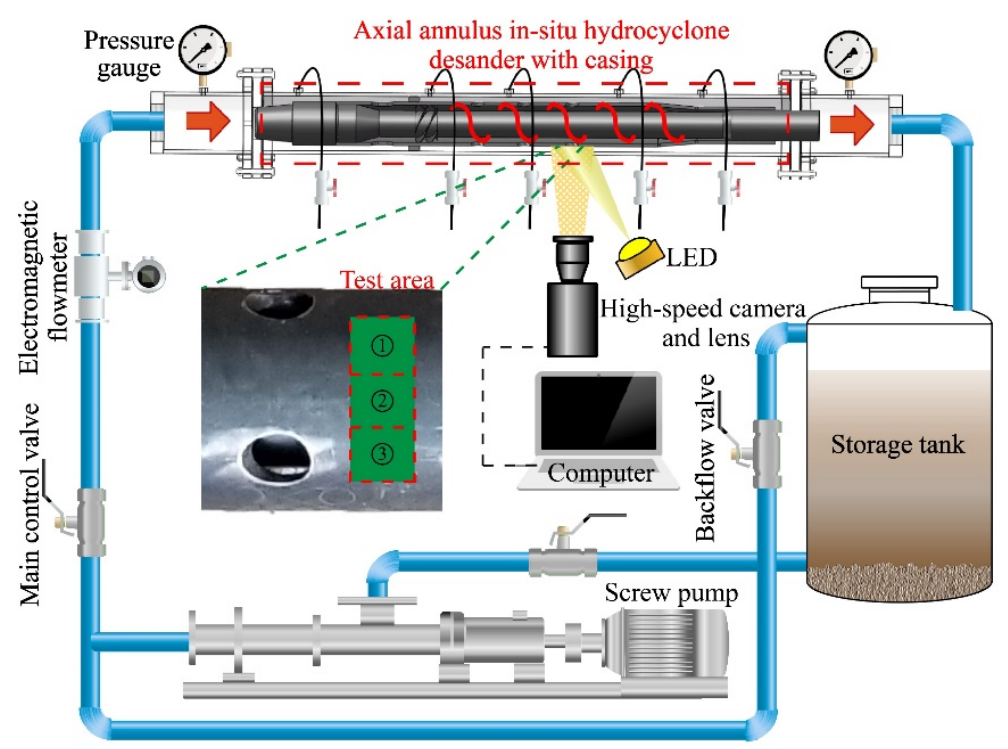

Figure 3. High-speed camera velocity measurement process.

\subsection{Analytical method}

\subsubsection{Split ratio.}

Using Image-pro plus to analyze and process the image after the velocity measurement. The horizontal movement velocity of the tracer particles at the sand outlet $U_{p s}$ is

$$
U_{p s}=\frac{f}{M}\left(\Delta x_{1}-\Delta x_{2}\right)
$$

Where $f$ is the frame frequency taken by the high-speed camera, fps; $\Delta x_{1}$ and $\Delta x_{2}$ are the horizontal distance between the initial position and final position of the tracer particle in the visible range and the reference object, respectively, $\mathrm{m} ; M$ is the number of image intervals corresponding to the first and last instantaneous positions of the tracer particles in the visible range.

The split ratio of the sand outlet $R_{u}$ is

$$
R_{u}=\frac{Q_{s}}{Q_{i}}=\frac{U_{p s} \cdot S_{a}}{Q_{i}}
$$

Where $Q_{s}$ is the volume flow rate from the sand outlet of the AAIHD, $\mathrm{m}^{3} / \mathrm{s} ; S_{a}$ is the cross-sectional area of the annulus between the AAIHD and the casing, $\mathrm{m}^{2}$.

\subsubsection{Separation efficiency.}

Take a sample from the sampling port on the casing for solid content test. And the solid content of quartz sand $C$ is

$$
C=\frac{m}{V}
$$

Where $m$ is the mass of solid particles, $\mathrm{mg} ; V$ is the volume of the suspension sample of quartz sand, $\mathrm{L}$.

The separation efficiency for sand $E_{s}$ is

$$
E_{s}=\frac{C_{s}}{C_{i}} \cdot R_{u} \times 100 \%
$$

Where $C_{i}$ is the solid content at the inlet, $\mathrm{mg} / \mathrm{L} ; C_{s}$ is the solid content at the sand outlet, $\mathrm{mg} / \mathrm{L}$.

\section{Results and discussion}

\subsection{Split ratio}

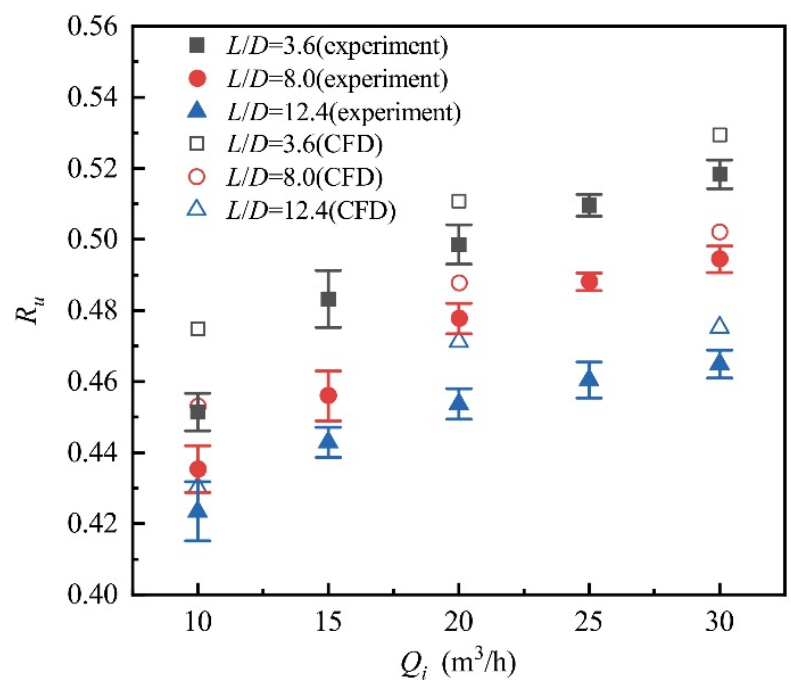

Figure 4. Split ratio.

The split ratio $R_{u}$ obtained by HSMA velocity measurement and CFD numerical simulation are shown in the figure 4 . The maximum error of the split ratio between the HSMA velocity measurement and CFD simulation is $5.2 \%$, which indicates that the split ratio measured by the HSMA is accurate. Split ratio obtained by the test increases with the increase of the flow rate. With increasing inlet flow rate, the rate of increase of the tested split ratio slows down and finally stabilizes. And split ratio obtained by the test gradually decreases with the increase of the separation zone. The split ratio obtained by simulation has the same changing trend as that obtained by experimental test. When $L / D=3.6$ and $Q_{i}=30 \mathrm{~m}^{3} / \mathrm{h}$, the tested maximum split ratio is 0.52 ; when $L / D=12.4$ and 
$Q_{i}=10 \mathrm{~m}^{3} / \mathrm{h}$, the tested minimum split ratio is 0.42 .
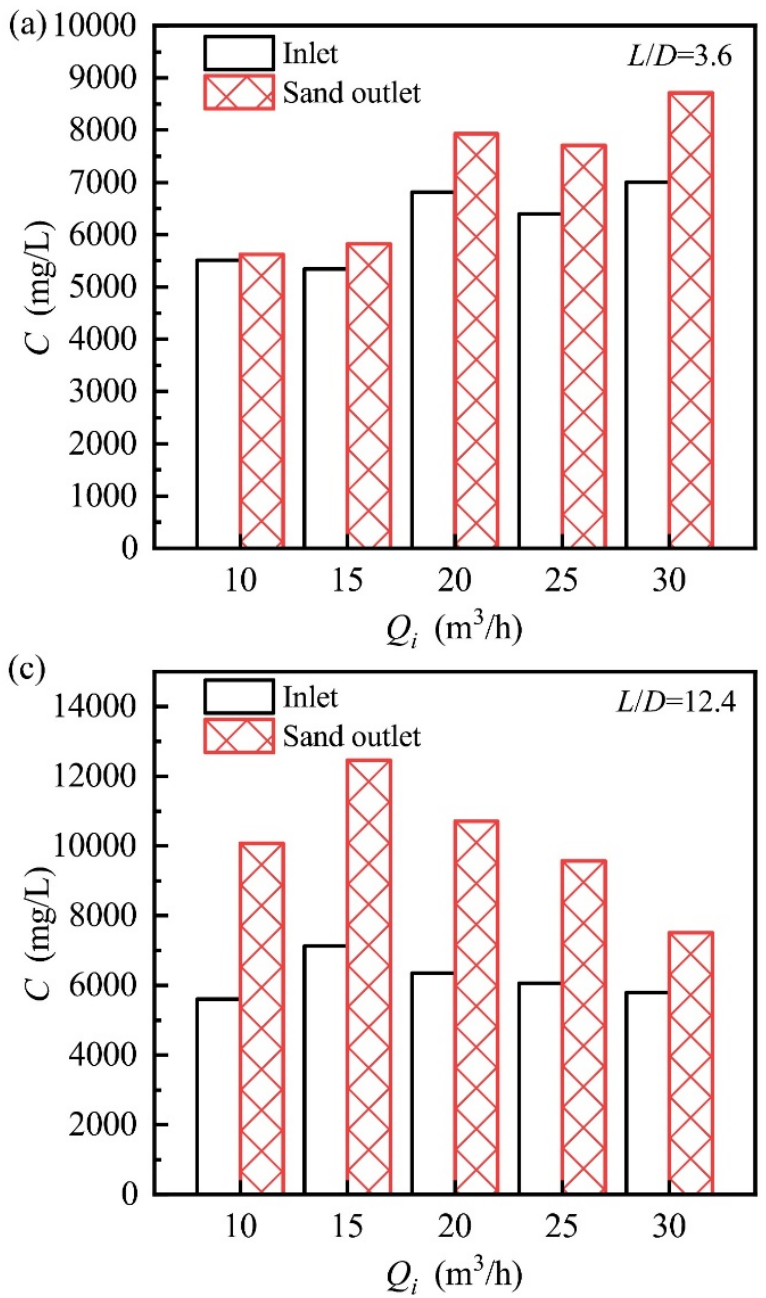

\subsection{Separation efficiency}

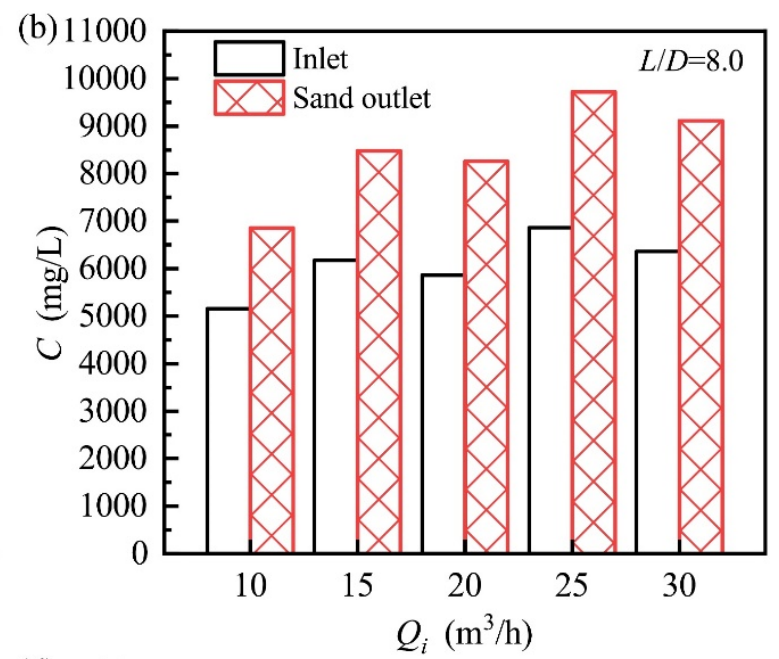

(d)

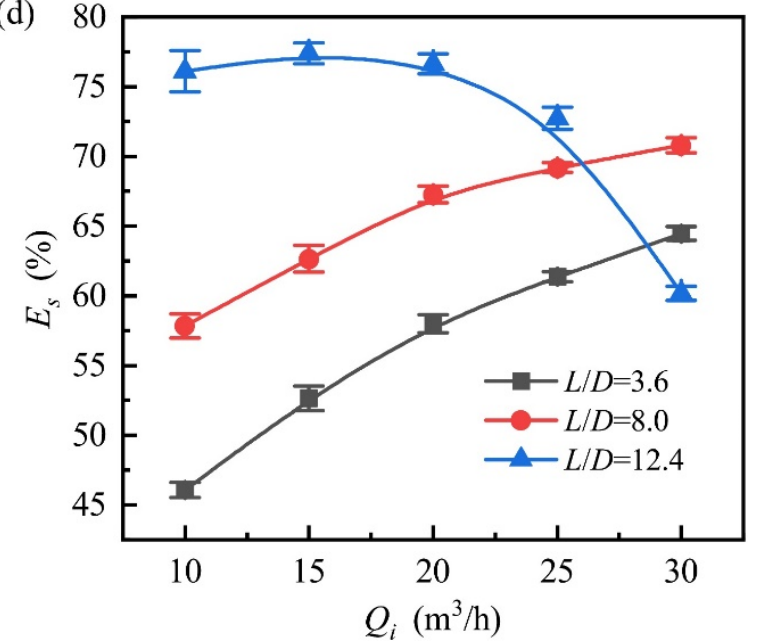

Figure 5. Solid content and separation efficiency. (a) Solid content of the inlet and sand outlet when $L / D=3.6$; (b) solid content of the inlet and sand outlet when $L / D=8.0$; (c) solid content of the inlet and sand outlet when $L / D=12.4$; (d) Separation efficiency of the AAIHD.

As shown in figure 5 (a), (b) and (c), the solid content of the inlet fluctuates between $5000-7000 \mathrm{mg} / \mathrm{L}$. When $L / D=3.6$ and $L / D=8.0$, the solid content of the sand outlet fluctuates with the increase of the inlet flow rate, but the overall trend is increasing, as shown in figure 5 (a) and (b). The maximum solid contents of the sand outlet corresponding to the $L / D=3.6$ and $L / D=8.0$ are $8711 \mathrm{mg} / \mathrm{L}$ and $9721 \mathrm{mg} / \mathrm{L}$, respectively, which are 1.3 times and 1.5 times the inlet solid content. It indicates that the sand removal effect of the AAIHD is remarkable. The separation efficiency also illustrates this point shown in figure 5 (d): when $L / D=3.6$ and $L / D=8.0$, the separation efficiency increases with the increase of the flow rate. The tangential velocity is larger as the separation zone is shorter [6], so that the radial resultant force on the particles has reached the separation requirement. In addition, the radial resultant force increases with the increase of the flow rate, which is conducive to the improvement of the separation performance. However, as shown in figure 5 (c), the solid content of the sand outlet increases first and then decreases rapidly while $L / D=12.4$. This is approximately the same as the shift trend of the separation efficiency reflected in figure 6 (d): when the inlet flow rate increases from $10 \mathrm{~m}^{3} / \mathrm{h}$ to $20 \mathrm{~m}^{3} / \mathrm{h}$, and the separation efficiency increases slightly and maintains to around $77 \%$; then the inlet flow rate increases to $30 \mathrm{~m}^{3} / \mathrm{h}$, the separation efficiency significantly decreases. Therefore, the capacity of the AAIHD should not be too high when the designed separation zone of AAIHD is longer.

The effect of the separation zone length on the separation efficiency can be simply analyzed by longitudinal comparison, as shown in figure 5 (d). The longer the separation zone, the higher the separation efficiency while the inlet flow rate range is $10 \mathrm{~m}^{3} / \mathrm{h}$ to 25 $\mathrm{m}^{3} / \mathrm{h}$. However, the extreme length of the separation zone is detrimental to separation efficiency when the inlet flow rate reaches $30 \mathrm{~m}^{3} / \mathrm{h}$. The reasons are as follows. The average residence time increases with the increase of the separation zone (as indicated in table 2), which is beneficial for separation. While the inlet flow rate is 30 $\mathrm{m}^{3} / \mathrm{h}$, the average residence time is the shortest during this flow rate. Furthermore, the excessively wide separation zone length means that the radial resultant force is very small, so the separation efficiency is low when $Q_{i}=30 \mathrm{~m}^{3} / \mathrm{h}$ and $L / D=12.4$. 


\section{Conclusion}

Aimed at the serious problem of sand production during solid fluidization mining of NGH in shallow layers of the deep sea, the axial annulus in-situ hydrocyclone desander (AAIHD) proposed in this paper has good separation performance. This research can provide a reference for the stable and safe exploitation of marine gas hydrates.

\section{Acknowledgements}

We would like to thank for the sponsorship of the National Key Research and Development Program of China (2019YFC0312305) and the Open Project of State Key Laboratory of Chemical Engineering (SKL-ChE-20C01).

\section{References}

1. Wang W, Wang X, Li Y, et al. (2020) Study on the characteristics of natural gas hydrate crystal structures during decomposition process. J. Fuel., 271: 117537.

2. Chang Y-L, Ti W-Q, Wang H-L, et al. (2021) Hydrocyclone used for in-situ sand removal of natural gas-hydrate in the subsea. J. Fuel., 285: 119075.

3. [3] Ma S H, Zheng J N, Tang D W, et al. (2019) Experimental investigation on the decomposition characteristics of natural gas hydrates in South China Sea sediments by a micro-differential scanning calorimeter. J. Applied Energy., 254: 8.

4. Yu T, Guan G, Abudula A. (2019) Production performance and numerical investigation of the 2017 offshore methane hydrate production test in the Nankai Trough of Japan. J. Applied Energy., 251: 113338.

5. Zhou S W, Chen W, Li Q P. (2014) The green solid fluidization development principle of natural gas hydrate stored in shallow layers of deep water. J. China Offshore Oil Gas., 26(5): 1-7.

6. Chen B, Ho K, Abakr Y A, et al. (2016) Fluid dynamics and heat transfer investigations of swirling decaying flow in an annular pipe Part 2: Fluid flow. J. International Journal of Heat and Mass Transfer., 97: 1012-1028. 